\title{
Sparse Odor Coding in Awake Behaving Mice
}

\author{
Dmitry Rinberg, ${ }^{1}$ Alex Koulakov, ${ }^{2}$ and Alan Gelperin ${ }^{1}$ \\ ${ }^{1}$ Monell Chemical Senses Center, Philadelphia, Pennsylvania, 19104, and ${ }^{2}$ Cold Spring Harbor Laboratory, Cold Spring Harbor, New York, 11724
}

Responses of mitral cells represent the results of the first stage of odor processing in the olfactory bulb. Most of our knowledge about mitral cell activity has been obtained from recordings in anesthetized animals. We compared odor-elicited changes in firing rate of mitral cells in awake behaving mice and in anesthetized mice. We show that odor-elicited changes in mitral cell firing rate were larger and more frequently observed in the anesthetized than in the awake condition. Only $27 \%$ of mitral cells that showed a response to odors in the anesthetized state were also odor responsive in the awake state. The amplitude of their response in the awake state was smaller, and some of the responses changed sign compared with their responses in the anesthetized state. The odor representation in the olfactory bulb is therefore sparser in awake behaving mice than in anesthetized preparations. A qualitative explanation of the mechanism responsible for this phenomenon is proposed.

Key words: olfactory bulb; in vivo recording; olfactory code; mitral cell; extracellular recordings; anesthesia

\section{Introduction}

A rose, by any other name, is represented by a spatiotemporal pattern of activity in the olfactory bulb and cortex (Lledo et al., 2005). The critical features of the rose odor representation are at present unknown. Information about volatile chemicals that comprise the odor of a rose is transferred from the olfactory receptors in the sensory epithelium to the next stage of processing: the olfactory bulb. Most of our knowledge about how the olfactory bulb works has been obtained from anesthetized preparations. Little is known about the properties of mitral cells, the first recipients of signals from receptors, in awake behaving animals. Some initial studies provided evidence that mitral cell behavior is different in the awake animal from what was expected based on studies of anesthetized preparations (Pager, 1983, 1985; Bhalla and Bower, 1997; Kay and Laurent, 1999).

Most of our present knowledge of odor representations in the olfactory bulb is based on mapping studies of glomerular activation patterns using a variety of techniques (Xu et al., 2000, 2003; Belluscio and Katz, 2001; Wachowiak and Cohen, 2001; Kauer, 2002; Bozza et al., 2004; Igarashi and Mori, 2005; Johnson et al., 2005; Salcedo et al., 2005). The link between glomerular activation patterns and responses of mitral cells has been studied in anesthetized preparations (Kashiwadani et al., 1999; Luo and Katz, 2001). This link clearly depends on behavioral state and should be established in the awake behaving animal (Kay and Laurent, 1999).

Mitral cell responses in awake behaving animals have been studied previously (Moulton, 1963; Pager, 1983, 1985; Bhalla and

Received Sept. 22, 2005; revised July 17, 2006; accepted July 18, 2006.

This work was supported by the Monell Chemical Senses Center, the Army Research Office, and The Whitehall Foundation. We thank Graeme Lowe and John Hopfield for comments on this manuscript and Fred Ollinger for technical assistance.

Correspondence should be addressed to Dmitry Rinberg, Howard Hughes Medical Institute, Janelia Farm Research Campus, Ashburn, VA 20147. E-mail: rinbergd@janelia.hhmi.org.

D01:10.1523/JNEUROSCI.0884-06.2006

Copyright $\odot 2006$ Society for Neuroscience $\quad$ 0270-6474/06/268857-09\$15.00/0
Bower, 1997; Kay and Laurent, 1999), as reviewed by Rinberg and Gelperin (2006). Several studies have shown how cellular and network responses of the olfactory bulb are affected by the nature and depth of anesthesia in acute preparations (Adrian, 1950; Nicoll, 1972; Stewart and Scott, 1976; Scott and Stewart, 1979; Fontanini and Bower, 2005). The main conclusion from these studies is that in the awake animal, spontaneous activity (Chaput and Holley, 1979) and odor responses are different from responses in anesthetized preparations and that mitral cell odor responsiveness is modulated by behavior (Kay and Laurent, 1999).

In addition, studies in several sensory processing and motor control pathways related to modalities other than olfactory have highlighted striking differences between cellular responses in the awake and anesthetized states (Schmidt and Konishi, 1998; Pack et al., 2001; Margrie et al., 2002; Movshon et al., 2003).

We recorded extracellularly the activity of single mitral cells or small ensembles of cells in the mouse olfactory bulb in both awake and anesthetized states and demonstrated striking differences between mitral cell responses recorded in these two states. These recordings were possible because of a novel microdrive implant technology (Fee and Leonardo, 2001), which allowed us to track activity of the same cell during its transition from the awake state to the anesthetized state and back. We show that spontaneous activity strongly increases in the transition from anesthetized to awake states. At the same time, cells that responded to odorants in the anesthetized state lost their odor sensitivity in the awake state. In several cases, odor responses in the awake state were weaker or of different sign compared with responses of the same cell in the anesthetized state. We focus only on firing rate responses, leaving other modes of olfactory information transfer for future studies. Our results suggest that the firing rate-based representation of odors in the olfactory bulb is sparser when the animal is in the awake state compared with the anesthetized state. 


\section{Materials and Methods}

A total of six male C57BL/6 mice were used in this study. Subjects were 6-8 weeks old at the beginning of behavioral training and were maintained on a $12 \mathrm{~h}$ light/dark cycle (lights on at 8:00 P.M.) in isolated cages in a temperature- and humidity-controlled animal facility. All behavioral training was conducted between 8:00 A.M. and 8:00 P.M. Mice had ad libitum access to food but were on a water restriction schedule designed to keep them at $80-85 \%$ of their baseline body weight. All animal care and experimental procedures were in strict accordance with a protocol approved by the Monell Chemical Senses Center Institutional Animal Care and Use Committee.

Extracellular signals from mitral cells in the mouse olfactory bulb were recorded in both awake behaving and anesthetized states. Waterdeprived mice were trained to poke their nose into an odor delivery port, wait until an odorant was delivered, and respond according to the training paradigm. The odor exposure time was controlled by the mouse for two behavioral paradigms and controlled by the experimenter for the third (BUZ) paradigm, as described below. Odor exposure time varied between 250 and $500 \mathrm{~ms}$. During one behavioral session, a mouse made from 100 to 500 trials and was exposed to a set of from two to eight different odorants. Immediately after some of the behavioral sessions, the mouse was anesthetized with ketamine/xylazine $(10 / 1 \mathrm{mg} / \mathrm{ml}, 0.01 \mathrm{ml} / \mathrm{g}$, i.p.) and gently placed into the same odor delivery apparatus with its nose in the odor port. We then compared responses of the same mitral cell with the same odorants recorded in the anesthetized state as recorded previously in the awake state.

All experiments were done in a modified Knosys (Lutz, FL) olfactometer (Bodyak and Slotnick, 1999). The combined odor-water port was replaced by an odor port and two separate water ports located to the right and left of the odor port, each water port at a distance of $35 \mathrm{~mm}$ from the center of the odor port. Photo emitters and detectors were installed in each port to monitor nose pokes into the port.

Behavioral paradigms and odor delivery. Mice were trained using one of three behavioral training paradigms. In each paradigm, the mouse initiated the trial by poking its nose into the odor port. The interruption of the photobeam in the odor port triggered a sequence of events. First, one of the eight pairs of odor valves was opened, and the final valve diverted the air flow away from the odor port. The air flow through the selected odorant vial was diluted 10 times by the main airflow stream and homogenized in the mixer chamber located just before the final valve. Some time later (randomly selected value between 0.5 and $1.5 \mathrm{~s}$ ), the final valve released and the prepared mixture of air and odorant was delivered to the odor port. The mouse sniffed the odorant and responded according to its previously learned association. In the "go-no-go" paradigm (GNG), the mouse was trained to run to a water port for the $\mathrm{S}+$ stimulus and get a water reward $(3 \mu \mathrm{l})$ or to withdraw from the port for the $S-$ stimulus and wait until the next trial could be initiated after a fixed intertrial interval, $3 \mathrm{~s}$ (two mice were trained in this paradigm). In the two-alternativechoice paradigm (2AC), the mouse learned to go only to the left or to the right water port in response to a given odorant (1 mouse).

To study olfactory responses of mitral cells in the absence of a learned association with the odorant, we developed a third behavioral paradigm (BUZ), in which the mouse needed to keep its nose in the odor port and be exposed to an odorant until an audio signal (buzz) sounded. If the mouse withdrew its nose from the port before the buzz, no water was available. If its nose was withdrawn after the buzz, the water reward was available independently of what odorant was presented or whether an odorant was presented. The BUZ paradigm allowed us to measure bulbar responses to novel odorants with no associative component. Three mice were trained in this paradigm.

The average time the mouse kept its nose in the port and was exposed to odorant in the GNG paradigm for S+ trials was $440 \mathrm{~ms}$ and for Strials was $570 \mathrm{~ms}$. In the $2 \mathrm{AC}$ paradigm, average odorant exposure time was $\sim 300 \mathrm{~ms}$. In the BUZ paradigm, the odorant exposure time was controlled by the experimenter. The buzz latency time was usually 500 $\mathrm{ms}$ after odorant onset, and the mouse withdrew its nose from the port $\sim 100$ ms after the beginning of the buzz, for a total odorant exposure time of $600 \mathrm{~ms}$.
To make odorant delivery as similar as possible in the anesthetized and awake states, the same sequence of events was repeated for the anesthetized mouse, but odorant delivery was triggered not by the mouse nose poke, but by the experimenter. The only substantial difference was that in the awake state the mouse withdrew its nose from the odor port and abruptly stopped odorant exposure, whereas in the anesthetized state it took a few seconds to wash out the odorant from the port by the flow of clean air.

We used multiple odorants (see Figs. 1-8 and supplemental material, available at www.jneurosci.org) obtained from Sigma-Aldrich (St. Louis, $\mathrm{MO}$ ). The odorants were stored in liquid phase (undiluted or diluted 1:10 in mineral oil) in dark vials. The odorant concentration delivered to the animal was one-tenth of the saturated vapor pressure attributable to air dilution. In several experiments, we used mixtures. The components were mixed in liquid phase in equal volume parts. (The full list of odorants and mixture components we used is in Table S1, available at www. jneurosci.org as supplemental material.)

Electrophysiology. After $\sim 2$ weeks of training, each animal was implanted with a motorized microdrive system that carried 3-9 sharp tungsten electrodes ( $3 \mathrm{M} \Omega$ at $1 \mathrm{kHz}$ impedance; Microprobes, Gaithersburg, $\mathrm{MD})$. The motorized microdrive device was similar to that described by Fee and Leonardo (2001). The position of each of the three shuttles was controlled remotely and independently. Each shuttle carried from one to three electrodes. The implantation was done under isoflurane gas anesthesia. The microdrive was installed so that electrodes were directed into the olfactory bulb at a $45^{\circ}$ angle in the sagittal plane. The main mass of the microdrive was carried by the posterior part of the skull. Mice could walk normally carrying a $3 \mathrm{~g}$ device shortly after recovery from anesthesia. The reference electrode was implanted through an additional small hole in a more posterior region of the skull. The common ground electrode was attached to one of the anchor screws. Five to seven days after implantation, electrodes were slowly moved into the olfactory bulb until action potentials from mitral cells were recorded. Approximate positions of the electrode tips are in the ventral anterior part of the bulb $\sim 1 \mathrm{~mm}$ from the midline. A schematic view of the microdrive and electrode positions is shown in Figure $1 F$.

After $5 \mathrm{~d}$ of recovery from surgery and $2 \mathrm{~d}$ of restricted water access, the mouse with implanted electrodes was put into the behavioral arena and connected to the microdrive motor controller and preamplifiers via a flexible cable. The behavioral events (photobeam status and valve signals) together with amplified electrophysiological signals were digitized (20 kHz sampling rate) using a data acquisition system (Nicolet Odyssey; LDS Test and Measurement, Middleton, WI) and analyzed off-line.

Cell search. The search for mitral cells was done in the awake state in a behaving freely moving mouse by moving the electrodes in the olfactory bulb with remotely controlled micromotors. The identity of mitral/ tufted cells was established by the following criteria. (1) Approximate stereotaxic coordinates of the ventral mitral cell layer (Paxinos and Franklin, 2003). The microdrive positioning system allowed rough monitoring of the absolute electrode displacement $(\sim 100 \mu \mathrm{m}$ accuracy) and fine control of sequential positions along a continuous electrode track $(\sim 1 \mu \mathrm{m}$ accuracy). (2) The signal from the mitral cell layer was localized in a narrow band of $100-120 \mu \mathrm{m}$ along the electrode movement axis (Pager, 1978; Kay and Laurent, 1999). This indicated the layered structure of the cells from which we were recording. (3) Mitral/tufted cells are larger than other cells in the bulb (Benson et al., 1984); thus, they produce larger and more stable spikes recorded by the extracellular electrodes (Buonviso et al., 2003). Occasionally, we transiently detected small spikes in the region corresponding to the granule cell layer. (4) The cells we analyzed had robust odor responses and typical mitral cell spontaneous activity patterns synchronized to respiration in the anesthetized state (Macrides and Chorover, 1972; Onoda and Mori, 1980; Chaput, 1986; Sobel and Tank, 1993; Buonviso et al., 2003) (Fig. 2A2,B2).

Electrodes were moved multiple times (10-20) forward and backward, up to $2 \mathrm{~mm}$ deep along the electrode path and back almost to the surface. The fact that we were able to find cells during each penetration, except during the few last runs, indicated that there was little damage to the mitral cell layer in the recording region. Some damage of the granule 
A

B

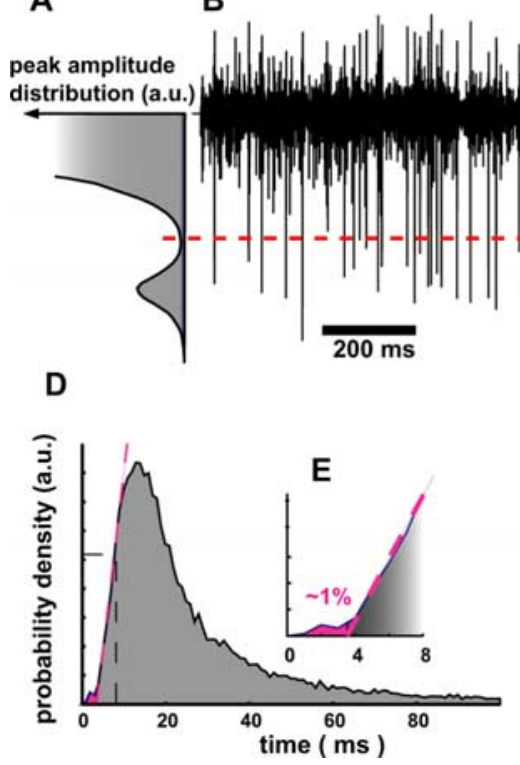

C
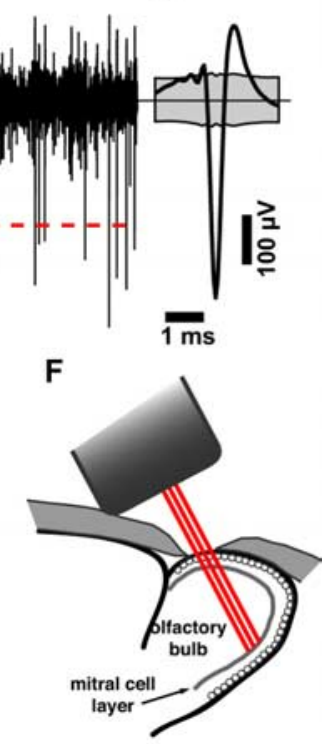

Figure 1. Extracellular recording from a presumptive mitral cell. $A$, Negative peak amplitude distribution. The red dashed line is a minimum in the peak amplitude distribution used as a threshold for spike detection. $\boldsymbol{B}$, Example of raw data with spikes. All peaks below the threshold (red dashed line) are considered to be spikes. $C$, Average spike shape. The gray area is the level of noise of $1 S D$ after subtraction of the averaged spike shape. $\boldsymbol{D}, \boldsymbol{E}$, Interspike interval distribution. The red dashed line is a linear fit of the rising edge of the distribution. The red area near zero is the proportion of spike intervals smaller than the absolute refractory period. The refractory period is estimated by the intersection of the red dashed line with the $x$-axis. $\boldsymbol{F}$, Approximate position of implanted electrodes in the mouse olfactory bulb. a.u., Arbitrary units.

layer was unavoidable; however, we did not see degradation or substantial changes of the mitral cell responses in each sequential penetration.

Spike identification and single-unit validation. The raw data were filtered off-line with a Butterworth six-pole filter, bandpass: $300 \mathrm{~Hz}$ to 3 $\mathrm{kHz}($ Fig. $1 B$ ). For a given epoch of recording, the distribution of the negative peaks was measured (Fig. $1 A$ ). The position of a minimum in the peak amplitude distribution was set as a threshold for spike registration. Every negative peak below this threshold was considered a spike. The average spike shape with SD margins for a typical recording epoch is shown in Figure $1 C$. Note that the SD at the peak of the spike is comparable with that of the prespike baseline. This means that the relatively wide distribution of the spike peaks was mainly caused by recording noise, not to spike variability. That was the first evidence for single-unit recording.

The spike detection criteria did not set any limitation on how close the spikes could be in time, as sometimes occurs for template matching methods; therefore, we were able to use the interspike interval distribution as an independent condition for single-unit validation (Fig. $1 D, E$ ). The intersection of the linear fit of the rising slope of the interspike interval distribution with the $x$-axis was taken as an estimate of the refractory period $(3.5 \mathrm{~ms})$. The presence of a refractory period was indicative of a single-unit recording. Less than $1 \%$ of interspike intervals fall into the range below the refractory period. Violation of the refractory interval by $1 \%$ of the events was caused by recording artifacts that were hard to avoid in behavioral recordings. In several cases, we observed such artifacts explicitly as large fluctuations in voltage with multiple closely spaced peaks producing interpeak intervals $<3.5 \mathrm{~ms}$.

Those recordings that had a minimum in the amplitude peak distribution were classified as single-unit recordings. For all of them, the violation of interspike refractory period was less then $1.5 \%$. A total of 11 sessions had well defined single-unit signals. Nine of 11 sessions had odor responses in the anesthetized state.

In cases in which the single-unit recording criteria were not satisfied, we treated the data as multiunit recordings. The threshold for spike detection was set at a level 5 times higher than the rms noise level in the recording. Such a stringent spike detection criterion often detected only part of the activity of the unit, i.e., only a portion of the spikes produced by one cell was detected. Although comparison of spontaneous firing rate in anesthetized and awake states was not meaningful in such sessions, the disappearance of odor responses during the transition from anesthetized to awake state could be established. We had eight sessions in which odor responses in the anesthetized state were observed in multiunit recordings.

Did we record from the same cell in the awake and anesthetized state? To answer this question for single-unit recordings, we tracked spike shape during the transition from the awake to anesthetized state and back (Fig. 3). Examples of raw data taken just before anesthesia (top row) and at various times after injection of anesthetic are shown in Figure $3 B$. The average firing rate went from $30 \mathrm{~Hz}$ to a few hertz during the first 5-6 min after anesthetic injection. The firing became bursty and synchronized with breathing, as observed visually. The shape of the negative peak distribution (Fig. $3 A$ ) remains the same as in the awake state. It had a bimodal shape, with the smaller peak corresponding to action potentials. However, the number of spikes per recording epoch decreased as the animal went deeper into the anesthetized state; the noise decreased, and the locations of the maximum and local minimum of the negative peak distribution slightly shifted toward lower amplitudes. The spike detection paradigm was the same as for the data before anesthesia. The average spike shape is shown in Figure $3 C$ (red line). For comparison, we also show the spike shape for the same unit in the awake state (thin black line) with the SD margins (gray area).

The spike shape slowly changed after injection of anesthetics. The average firing rate quickly decreased and then slowly increased. The average spike shape became slightly smaller and wider. However, the changes were slow, with no noticeable abrupt changes. The overall picture was consistent with the interpretation that we were recording from the same unit both in awake and anesthetized states.

Analysis of odor responses. For 26 cells, we compared responses to multiple odorants (from two to eight for one cell) in awake and anesthetized states. (For the full list of odorants, see Table S1, available at www.jneurosci.org as supplemental material.)

In the anesthetized state, we measured the distribution of firing rates in $0.5 \mathrm{~s}$ bins without odorant presentation and compared it with odorant-elicited firing rates measured in the interval from 0.5 to $1 \mathrm{~s}$ after odorant onset. We
Figure 2. Raw traces of extracellular recordings from the same location in an awake, behaving $(\boldsymbol{A} \mathbf{1}, \boldsymbol{B} \mathbf{1})$, and anesthetized $(\boldsymbol{A} \mathbf{2}$ B2) mouse for two odorants: citral $(\boldsymbol{A} 1, \mathbf{A} \mathbf{2})$ and amyl acetate $(\boldsymbol{B} 1, \mathbf{B} 2)$. The solid horizontal bars under each trace indicate time of odorant exposure. The dashed bars indicate the time of final valve activation that in the behavioral paradigm corresponds to the time the mouse spent in the port before odor delivery. 
consider the cell responding to odorant if an ANOVA test gave $p<0.05$ for the hypothesis that the distributions of spike counts in $0.5 \mathrm{~s}$ intervals during odorant presentation over several trials had the same mean value as the mean spontaneous firing. The odorant response in the awake state was weaker than in the anesthetized state. To test whether a cell had a response to an odorant in the awake state we compared multiple trials for two or more odorants, one of which did not produce a response in the anesthetized state, and compared the spike counts for the last $300 \mathrm{~ms}$ before mouse nose withdrawal from the odor port. We considered a cell responsive to an odorant if an ANOVA test gave $p<0.05$ for the hypothesis that the distributions of spike counts for responses to the odorants had the same mean value.

We synchronized all recording trials by the moment of nose withdrawal from the odor port for averaging mitral cell responses across trials. The release of the final valve and subsequent odorant delivery occurred at a random time after a mouse poked its nose into the odor port, and thus at a random phase of the mouse breathing cycle. This is why odorant delivery to the odor port did not lead to immediate access of odorant molecules to the olfactory epithelium. As shown by Uchida and Mainen (2003), rats withdrew their noses from the odor port at the same phase of the breathing/sniffing cycle. We assumed that physiological and information processing states of the mouse at the moment of nose withdrawal from the odor port were more similar than at the moment of odorant delivery triggered by an external valve.

For single-unit recordings, we made a direct comparison of odorant responses in awake and anesthetized states. In multiunit recordings, we assumed that we recorded partial signals from one or a few cells. If an ensemble of cells responded to an odorant in the anesthetized state, that meant that at least one cell in the ensemble was odorant sensitive. The absence of an odorant response in the awake state meant that none of the recorded cells from the ensemble was responsive to the odorant. If the response to an odorant was observed in both awake and anesthetized states, we could not exclude the possibility that two different cells responded to the same odorant in two states. However, the chances of such an event were relatively low, because the probability of response to an odorant in the awake state was low. Also, in single-unit recordings, we have not observed a cell that did not respond to an odorant in anesthetized state but did respond to the odorant in the awake state.

\section{Results}

In 26 sessions we recorded extracellular signals from presumptive mitral/tufted cells in both awake and anesthetized states. Eleven of 26 were classified as single-unit recordings. Typical raw traces for single-unit recordings in awake and anesthetized states are shown in Figure 2. Each panel presents two traces for awake (Fig. $2 A 1, B 1)$ and anesthetized $(A 2, B 2)$ states showing responses to one of two odorants: citral (CIT) $(A)$ or amyl acetate $(\mathrm{AA})(B)$. In the awake state the average mitral cell prestimulus firing rate for this cell was $\sim 30 \mathrm{~Hz}$, whereas in the anesthetized state the baseline firing rate was $\sim 9 \mathrm{~Hz}$. There was no clear response to either

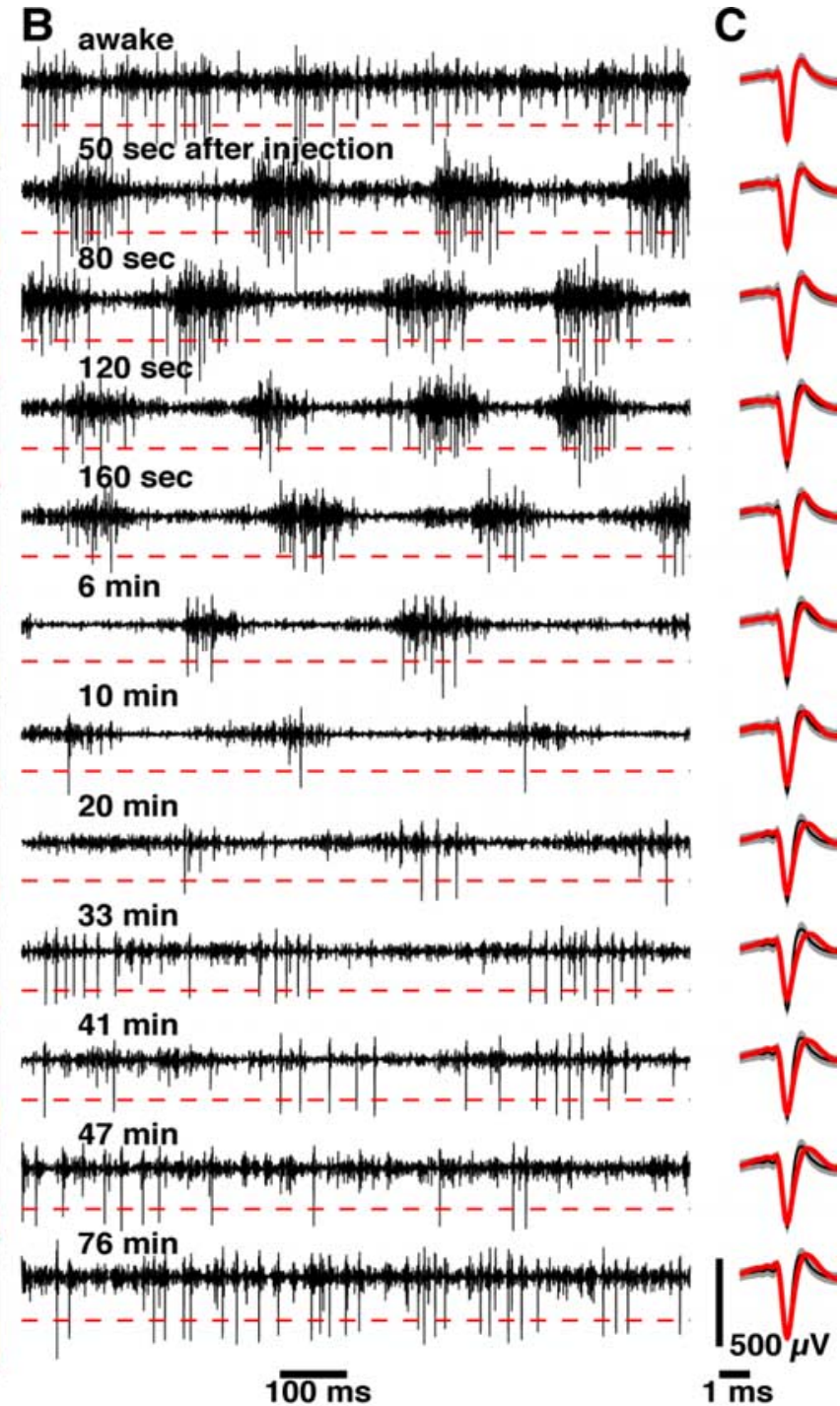

$1 \overline{00 \mathrm{~ms}}$

Figure 3. Tracking of peak amplitude distribution and spike shape in the transition from the awake to anesthetized state and back. Each raw data trace corresponds to a certain time relative to anesthetic injection. The first raw data trace is taken in the awake is the area below spike detection threshold defined as a local minimum in peak amplitude distribution. The data shown in the mplitude histograms were collected in a 20 s window bracketing the time shown in the raw data traces. The peak counts are no of $1 \mathrm{~s}$ of raw data. The dashed red line is the threshold for spike detection. $\boldsymbol{C}$, Thick red line is the averaged spike shape in the $20 \mathrm{~s}$ window. A thin black line with gray margins is the average spike shape in the awake state. Margins are 1 SD.

odorant in the awake state, whereas we observed a strong response to AA in the anesthetized state (Fig. 2B2).

For the same cell, firing rates averaged over multiple trials for awake and anesthetized states are shown in Figure $4 \mathrm{~A}$. In the awake state, each odorant was presented 113 times, whereas in the anesthetized state each odorant was presented 11 times.

We find consistently that the spontaneous intertrial firing rate in the awake state is higher than that recorded in the anesthetized state. The summary for 11 single-unit recordings in which we were able to compare spontaneous firing rates in the awake and anesthetized states is presented in Figure $5 \mathrm{~A}$. Spontaneous firing rate in the awake state is plotted versus spontaneous firing rate in the anesthetized state. All of the points lie above the diagonal line (Fig. 5A, dashed line).

In the awake state, in 10 of 26 recordings we observed modulation of the average firing rate by behavior. The increase of firing 


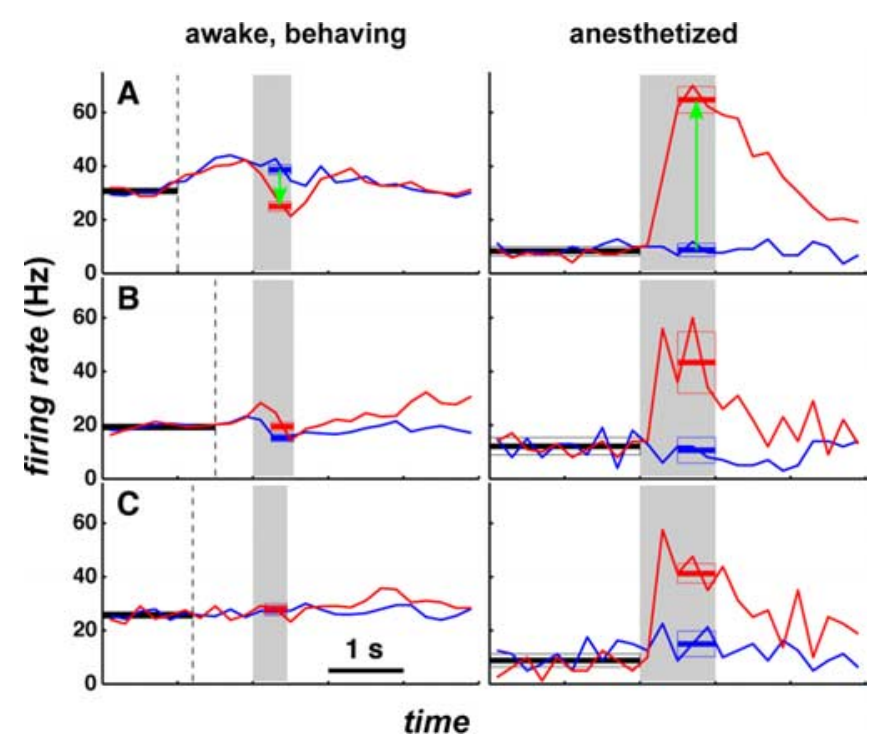

Figure 4. Averaged firing rate responses of the same mitral cells to odorants in anesthetized (right panels) and awake states (left panels). The gray area is the time interval of odorant exposure. $\boldsymbol{A}$, Inhibitory response in the awake state. 0 dorants: red, amyl acetate $(113,11)$; blue, citral $(113,11) . \boldsymbol{B}$, Excitatory response in the awake state. Odorants; red, 2-nonanone $(76,5)$; blue, amyl acetate $(69,5)$. C, No response to odorant in the awake state. Odorants: red, amyl acetate $(62,3)$; blue, citral $(60,3)$. The numbers after each odorant are the number of trials in the awake and anesthetized states, respectively. The vertical dashed line in the left panels is the average time a mouse poked its nose into the odor port. Solid thick lines with thin line margins indicate average firing rates with SEs and time intervals at which they were measured for spontaneous activity (black), null response (blue), and response to an odor (red). The data for all panels are from putative single-unit recording sessions.

rate occurred before odorant presentation during nose poke into the odor port (Fig. $4 A, B$ ). A similar phenomenon was also reported in previous work by Kay and Laurent (1999). Modulations in firing rate before odorant sampling were observed for GNG and 2AC behavioral paradigms but not for the BUZ paradigm.

Because of odorant-independent behavioral modulations of the firing rate in the awake state, we chose to measure the relative odorant responses for two odorants. For a single odor we could not unambiguously classify the odorant response, independent of the behavioral modulatory response, as excitatory or inhibitory. Thus, in Figure $4 \mathrm{~A}$ during odorant presentation in the awake state, the firing rate decreased from the level immediately before odor onset in response to both AA and CIT, but for AA (red trace) the decrease was larger than that for CIT. One could assume no odorant response and only behavioral modulation to AA and a weak excitatory response to CIT. Conversely, one could assume an inhibitory response to AA and no response to CIT. To resolve this ambiguity, we define a "null" response in both states to be the response to an odorant or a set of odorants that in the anesthetized state produces no significant change in firing rate compared with the preodorant spontaneous activity. We measured responses to other odorants in the awake state relative to a null response. Thus, in Figure $4 A$ the responses to CIT (blue line) in both the anesthetized and awake states are considered null responses. Responses to AA relative to the null responses are shown by green arrows. The arrows indicate the extent of the differences between the null response, i.e., the firing rate during CIT presentation, and the response to AA. Note that the response to AA relative to the null response was strongly excitatory in the anesthetized state and modestly inhibitory in the awake state.

Similar inhibitory responses in the awake state were observed in 3 of 26 cell-odorant pairs. Four cell-odorant pairs demon-

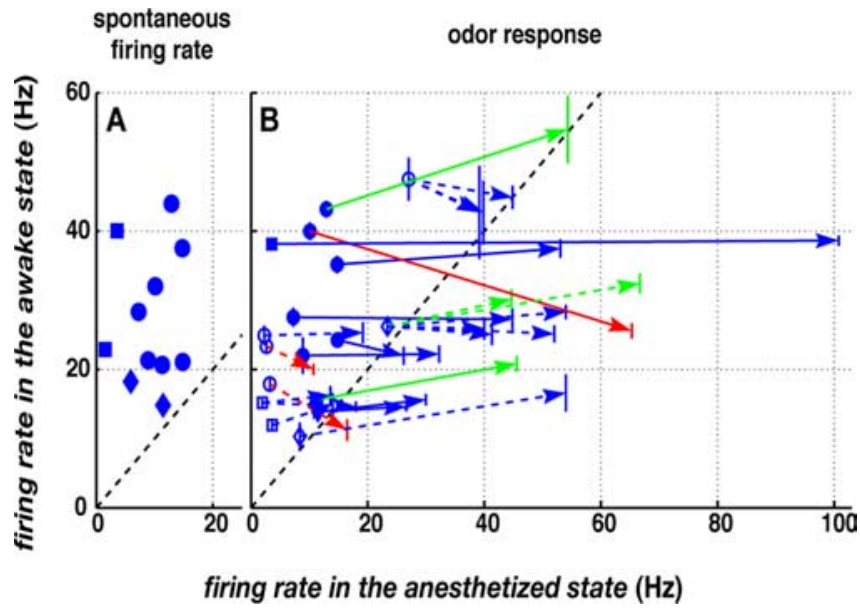

Figure 5. Summary of all recordings. $\boldsymbol{A}$, Spontaneous firing rate for single-unit recordings in the awake state versus spontaneous firing rate in the anesthetized state during the first $10 \mathrm{~min}$ after anesthesia. Error bars are smaller than the symbols. $\boldsymbol{B}, 0$ dorant responses for single units (filled symbols and solid lines) and multiunit recordings (open symbols and dashed lines). Each arrow starts at the null responses for a given cell and ends at the response to an odorant. Vertical lines at the origin and the end of each arrow are SEs for firing rate estimations in the awake state. For blue arrows, there are no statistically significant differences $(p>0.05)$ between the null response and odorant response. Green and red arrows indicate recordings for which there is a statistically significant excitatory (green) or inhibitory (red) odorant response ( $p<0.05$ ). Symbols in $\boldsymbol{A}$ and $\boldsymbol{B}$ indicate the type of behavioral paradigm used in the recording: circle, GNG paradigm; square, 2AC paradigm; diamond, BUZ paradigm.

strated excitatory responses in the awake state; two of them were recorded from single units. An example of a single-unit excitatory response in the awake state is shown in Figure $4 B$. Most frequently we observed no response to an odorant in the awake state that elicited a strong excitatory response in the anesthetized state: this pattern was found for a total of 19 cell-odorant pairs, of which 7 were single-unit recordings. An example of no response in the awake state to an odorant producing a clear excitatory response in the anesthetized state is shown in Figure $4 C$.

We present responses of all cell-odorant pairs in Figure $5 B$ as arrows in the coordinates of firing rate in the awake state versus firing rate in the anesthetized state. The arrows originate at null responses in both awake and anesthetized states for a given cell to a given odorant and end at responses of the same cell in both states to a different odorant. A total of 26 arrows is shown for 17 cells. The symbol at the origin of each arrow corresponds to the behavioral paradigm used during collection of the data. Filled symbols and solid lines correspond to single-unit recordings, whereas open symbols and dashed lines correspond to multiunit recordings. Green arrows correspond to significant excitatory odorant responses in the awake state, and red arrows correspond to significant inhibitory responses in the awake state. The significance of responses was established by statistical comparison of firing rate distributions for odorant responses and null responses. SEs of firing rate estimations in the awake state are shown by vertical bars near the origin and the end of each arrow. A summary for all recordings is presented in Table 1.

All of the origins of the arrows lie above the diagonal, signifying that the firing rates for null responses in the awake state, which were close to the spontaneous firing rates, were always higher than firing rates for null responses in the anesthetized state. Most of the arrows are parallel or close to parallel to the horizontal axis, showing that odorant responses in the anesthetized state were larger than in the awake state. The histogram of the slopes of the arrows is presented in Figure 6. The slope of the 
arrow is the ratio between odorant responses in the awake and anesthetized states. The range of observed ratios is from -0.5 to 0.3 .

We also examined the time course of anesthetic action in five sessions using two mice. Typical data are presented in Figure 7. Just after the injection of anesthetics in the first $10-20 \mathrm{~s}$, the spontaneous firing rate increased, presumably because of transient neural activation by the anesthetic injection or fast mouse movements, and then dropped to a very low level of a few hertz during 3-4 min. As the anesthetic wore off, the spontaneous firing rate slowly increased until it reached the typical awake level (Fig. 7, filled circles). The amplitudes of odorant responses evolved in the opposite direction. We measured the odorant response after establishing a deep level of anesthesia. The average firing rate measured during the first $2 \mathrm{~s}$ after odorant onset slowly decreased while the anesthetic wore off (Fig. 7, open circles). Fifty to sixty minutes after the injection of anesthetic, the animal started moving, at which point, based on a few measurements, we could not distinguish an odorant response from spontaneous firing. Although measurements of spontaneous firing rate just after the injection of anesthetics were done only in a few anesthesia sessions, the overall patterns and characteristic time scales of spontaneous firing rate and odor responses while the anesthesia wore off were the same in all experimental sessions.

Most of the data (20 of 26 sessions) were obtained when the mouse performed two or more odorant discrimination tasks (GNG or 2AC paradigms). Thus, the responses to an odorant might be modulated by an odorant association, such as water availability for the $\mathrm{S}+$ odorant and no water available for the Sodorant (GNG) or the location of the water at the left or right ports (2AC). For example, in both cases shown in Figure 4, $A$ and $B$, odorants that produced a higher firing rate during odorant presentation corresponded to the $\mathrm{S}+$ stimulus, i.e., odorants that were rewarded. The obvious question is whether the responses to an odorant in the awake state correspond to the odorant chemical composition or the odorant association, e.g., S+ or S-. To answer this question we developed a behavioral paradigm during which the mouse samples odorants without any association between the odorant and other cues or rewards (BUZ paradigm; see Materials and Methods). In one session using the BUZ paradigm, we observed both excitatory and inhibitory mitral cell responses to different odorants (Fig. 8). This observation showed that both inhibitory and excitatory mitral cell responses could be obtained for odor stimuli without association with reward.

\section{Discussion}

In this study, we show that responses to odorants are weaker in awake behaving animals compared with anesthetized animals, although at the same time the spontaneous firing rate is higher in the awake state than in the anesthetized state. This observation includes both smaller amplitudes of firing rate modulation and an overall reduction in the number of cells displaying odorant sensitivity (sparsening) in the awake state. Of 17 cells that responded to 26 odorants in the anesthetized state, only 6 showed responses to 7 odorants in the awake state; 3 of these 7 changed the sign of their response between the anesthetized and awake states.

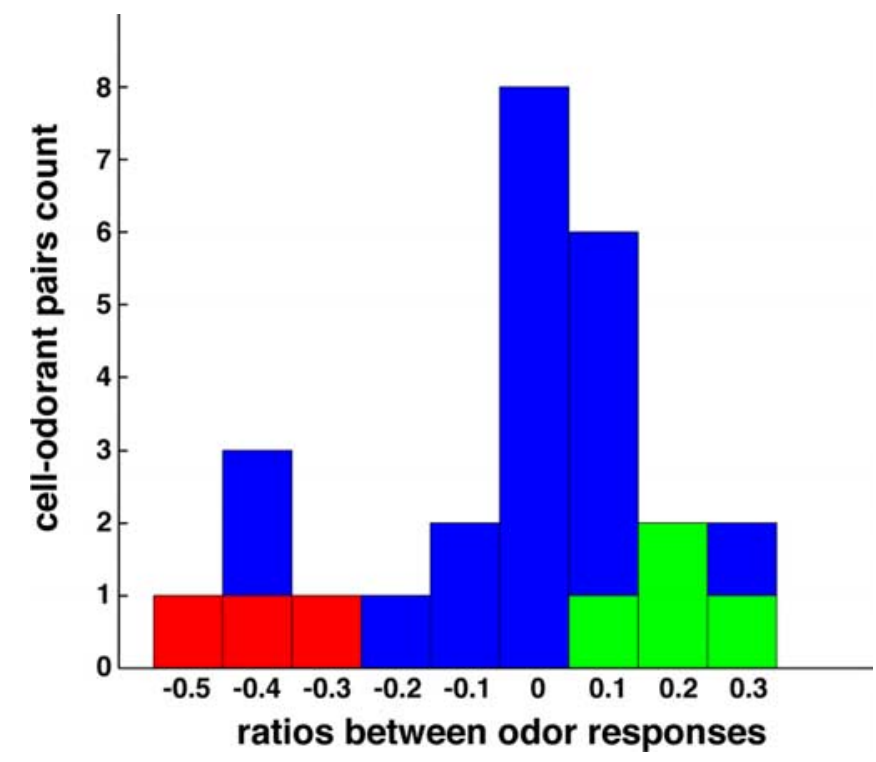

Figure 6. Histograms of ratios between odorant responses in awake and anesthetized states (slopes of the arrows in Fig. 5B). Green/red bars are the number of statistically significant odor responses $(p<0.05)$ for excitatory/inhibitory responses, which correspond to green/red arrows in Figure $5 B$.

\section{Experimental biases}

We recorded from cells that have nonzero spontaneous activity in the awake state. Silent mitral/tufted cells may exist in the awake state. However, during odorant presentation, we never observed the appearance of new units in either the awake or anesthetized states.

We recorded from a small percentage of the $\sim 50,000$ mitral/ tufted cells in a mouse olfactory bulb and used a small set of odors. This set may not include the most effective stimuli that drive these cells. However, based on our analysis we do not make any conclusions about absolute odor sensitivity in awake and anesthetized states, but we present only comparative analyses for cells excited by odors in the anesthetized state.

We limited ourselves to the use of only the safest injectable anesthetics, ketamine/xylazine, to minimize risks to the health of the trained mouse with implanted electrodes. Other anesthetics may have different pharmacological effects. However, the overall picture of mitral cell responses in the anesthetized state in our studies is consistent with other studies that used different anes- 


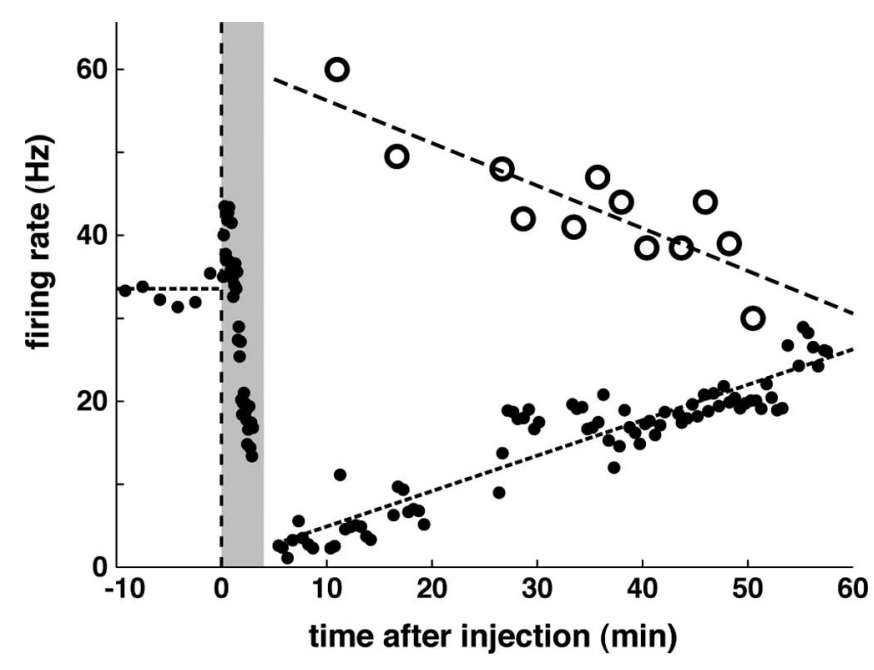

Figure 7. The spontaneous firing rate (small filled circles) averaged during 30 s as a function of time after anesthetic injection. The gray area is the time interval of the transition from the awake state to deep anesthesia. During this time interval, firing rate was averaged during $5 \mathrm{~s}$ intervals. Large open circles are the average firing rate during 2 s intervals after onset of odorant (amyl acetate) presentation. Dashed lines are linear fits used as a guide.

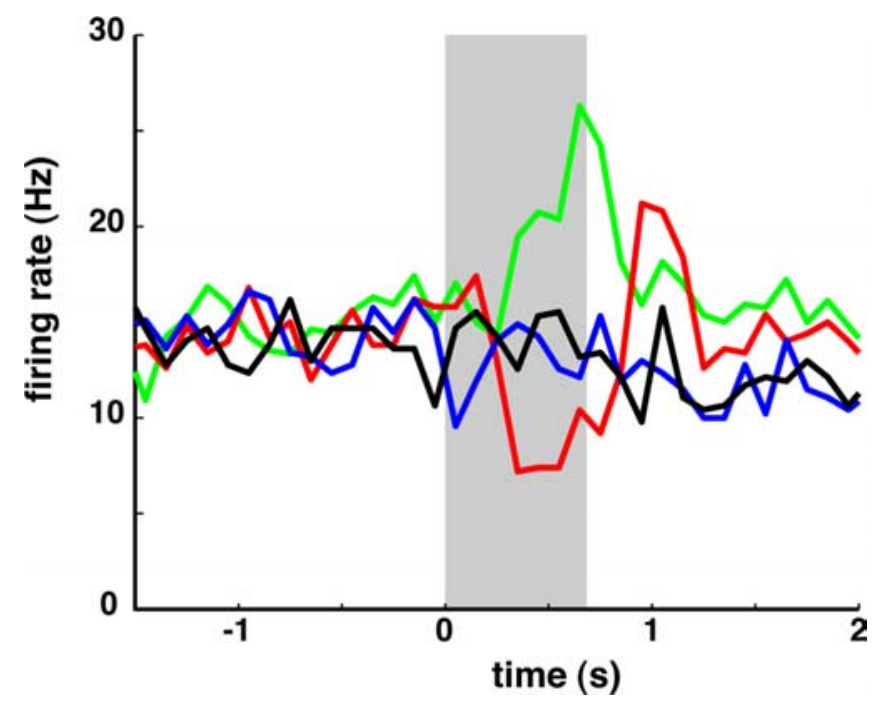

Figure 8. Average firing rate in response to multiple presentations of different odorants without learned associations between odorant presentation and water presentation (BUZ paradigm; see Materials and Methods). Black line, no odorant (54); blue line, mix 5 (50); red line, mix 3 (47); green line, mix 6 (47). Numbers after each stimulus are the number of trials.

thetics: Nembutal (Wellis and Scott, 1990) and urethane (Sobel and Tank, 1993; Nagayama et al., 2004). Most of the previous studies observed relatively low spontaneous firing rates of mitral/ tufted cells in the anesthetized state. Mitral cell activity in the anesthetized state has a pronounced periodic pattern that is synchronized with breathing (Sobel and Tank, 1993; Fontanini and Bower, 2005). The same pattern is also seen in our data (Fig. $2 A 2, B 2)$.

\section{Possible neural mechanisms}

Our observations raise the following question: how does the action of anesthetics cause two opposite effects, decrease of spontaneous firing rate and increase of odor responsiveness? We argue that the results presented here could be explained if two effects of anesthesia are assumed: (1) decrease in the odor-independent input current into mitral/tufted cells and (2) overall decrease in the recurrent inhibition.

A decrease of input current could be attributable to the block of NMDA receptors that mediate transmission from receptor cells. However, this explanation appears unlikely, because reduction of NMDA transmission in urethane-anesthetized rats by various NMDA receptor antagonists does not lead to noticeable changes in the mitral cell spontaneous firing rate (Wilson et al., 1996). It is also true that anesthetics that have not been reported to block NMDA receptors, such as urethane, yield similar reductions in mitral cell spontaneous rate (Pager, 1983; Motokizawa, 1996; Kay and Laurent, 1999). We argue therefore that modulatory centrifugal inputs that are sensitive to the animal's state of vigilance, such as serotonin-containing fibers from the dorsal raphe nucleus and cholinergic fibers from the basal forebrain (Shipley and Ennis, 1996; Haberly, 2004), could modulate mitral cell spontaneous activity. Serotonergic inputs target the glomerular cell layer and exhibit effects on juxtaglomerular cells and directly on a subset of mitral cells (Hardy et al., 2005). The cholinergic centrifugal projections to the bulb terminate primarily onto interneurons (Nickell and Shipley, 1988; Le Jeune and Jourdan, 1993, 1994; Kasa et al., 1995). Experiments in slice preparations have demonstrated a possible direct effect of acetylcholine (ACh) on mitral cells, suggesting an extrasynaptic diffusion of ACh (Castillo et al., 1999). Interestingly, the spontaneous firing rate in these slice experiments has been found to be $\sim 5.5 \mathrm{~Hz}$, even when fast glutamatergic and GABA-mediated (GABAergic) conductances were blocked. Application of carbachol, a cholinergic receptor agonist, increased the spontaneous firing of mitral cells in slice preparations to an average of $28 \mathrm{~Hz}$, which is similar to our measurements of spontaneous mitral cell activity in the awake state (Table 1). Increased cholinergic inputs in the awake state could be provided by activity of cholinergic neurons of the basal forebrain (Manns et al., 2000; Lee et al., 2005). These findings suggest that state-dependent cholinergic modulation of mitral cell spontaneous firing could account for the magnitude of the effect we observed, although other mechanisms are also possible.

The increase in the mitral cell odorant-evoked response in the anesthetized case could be attributable to a reduction in the strength of mitral recurrent inhibition. The latter may be caused by dendro-dendritic connections with granule cells or feedback from corticobulbar projections (Shipley and Ennis, 1996; Haberly, 2004). Both possibilities are qualitatively consistent with the results presented here. Indeed, because mitral cells have excitatory projections onto granule cells and receive inhibitory inputs from granule cells, the recurrent feedback loop controlling the odor response has an overall inhibitory effect. Application of anesthetic may decrease the strength of recurrent inhibition in this inhibitory loop by either direct block of the NMDA receptors mediating synaptic transmission between mitral and granule cells (Wilson et al., 1996) or by state-dependent suppression of GABAergic conductances (Castillo et al., 1999). Reduction of inhibition is expected to lead to a more vigorous odorant response in the anesthetized state. A more quantitative description of the reduction of odorant-evoked responses by changes in mitral cell inhibition is given in the supplemental material, available at www.jneurosci.org.

\section{Implications for olfactory code}

In this study, we show that the olfactory bulb generates stronger responses to odorants, both by number of responding mitral cells 
and amplitude of mitral firing rate changes, in the anesthetized state.

The reduction of the number of cells responsive to odorants in the awake state, compared with the anesthetized state, could be explained by an overall weakening of the odor response. The odor response in the awake state is weak, and averaging responses over more trials may result in a larger number of cell-odorant pairs, which have statistically significant odorant responses. The response may be weak but not sparse, meaning that the cells do not lose their odor sensitivity but rather their responses become very weak. However, there are two arguments for sparseness of the olfactory code in the awake state. First, a weak response may be considered to be no response, because in the behavioral situation an animal needs to make a decision based on only a few trials of odorant presentation, the animal cannot use averaging across multiple trials to improve signal-to-noise ratio and extract weak signals. The second argument is based on the shape of the histogram of all observed ratios between odor responses in awake and anesthetized states (Fig. 6). Although the statistical significance of the odorant responses at both edges of the histogram (green and red areas) may change with increasing the number of trials, the overall shape does not depend on the number of trials to a first approximation. The peak at zero indicates that most of the cells not only have a relatively low odorant response in the awake state but favor zero response. For these cells, the odorant response is weak; thus, the code becomes sparse.

The reduction in the number of mitral cells responding to odorants and weakening of odorant sensitivity make an olfactory firing rate code less reliable in the awake state. Our data suggest that the signal-to-noise ratio becomes weaker, and the amount of odorant information transmitted by the odorant-sensitive cells becomes lower in the awake state. It is usually assumed that a neural code is optimized for representing information about the stimulus in the most efficient manner (Barlow, 1961). That mitral cells seemingly display a more reliable odor response in the anesthetized animal suggests that factors other than representation efficiency determine mitral cell responses in the awake state.

One of the hypotheses discussed in the literature (Lennie, 2003; Olshausen and Field, 2004) is that sparse codes minimize metabolic costs. This explanation does not appear satisfactory here, because the spontaneous rate is actually higher in awake animals. Brody and Hopfield (2003) proposed that olfactory information is carried by the relative spike timing of mitral cells. The increased spontaneous rate in the awake state should facilitate this method of information transmission. In the relative timing framework, the modulations of firing rate by behavior and olfactory stimuli observed here are secondary to the precise action potential timing. This possibility is difficult to rule out, because the specific group of cells synchronizing their responses to a given odorant is almost impossible to find in a limited set of recordings.

Another possibility is that mitral cell activity multiplexes odor responses with behavioral variables. In many of our behavioral sessions, we observed robust modulations of mitral cell firing by anticipation of stimulus delivery (Fig. 4). The anticipatory response is usually excitatory and may send an important signal, which facilitates odorant recognition. Increased baseline firing may render both inhibitory and excitatory responses possible. Because of a considerable reduction in the number of responsive cells in the awake state, our results suggest that mitral cells represent information in a substantially different way from the ORNs. Multiplexed with behavioral modulations, mitral/tufted cell responses may convey odorant information that is relevant to the behavior associated with the odorant. This factor may differentiate activity in the bulb between awake and anesthetized cases.

In conclusion, we show that odor representations by mitral cells of the awake behaving mouse are sparser than representations observed in the anesthetized mouse. The number of cells responsive to odorants and the amplitude of odorant responses are decreased in the awake condition compared with the anesthetized state. We propose that a change in recurrent inhibition is responsible for this difference. We suggest that in the awake animal, the output of the olfactory bulb represents the integration of both odor stimuli and behavioral variables, relevant to odor expectation, discrimination, context, and predictive associations.

\section{References}

Adrian ED (1950) The electrical activity of the mammalian olfactory bulb. Electroencephalogr Clin Neurophysiol 2:377-388.

Barlow HB (1961) Sensory communication. Cambridge, MA: MIT.

Belluscio L, Katz LC (2001) Symmetry, stereotypy, and topography of odorant representations in mouse olfactory bulb. J Neurosci 21:2113-2122.

Benson TE, Ryugo DK, Hinds JW (1984) Effects of sensory deprivation on the developing mouse olfactory system: a light and electron microscopic, morphometric analysis. J Neurosci 4:638-653.

Bhalla US, Bower JM (1997) Multiday recordings from olfactory bulb neurons in awake freely moving rats: spatially and temporally organized variability in odorant response properties. J Comput Neurosci 4:221-256.

Bodyak N, Slotnick B (1999) Performance of mice in an automated olfactometer: odor detection, discrimination and odor memory. Chem Senses 24:637-645.

Bozza T, McGann JP, Mombaerts P, Wachowiak M (2004) In vivo imaging of neuronal activity by targeted expression of a genetically encoded probe in the mouse. Neuron 42:9-21.

Brody CD, Hopfield JJ (2003) Simple networks for spike-timing-based computation, with application to olfactory processing. Neuron 37: $843-852$.

Buonviso N, Amat C, Litauson S, Roux S, Royet J-P, Farget V, Sicard G (2003) Rhythm sequence through the olfactory bulb layers during the time window of a respiratory cycle. Eur J Neurosci 17:1811-1819.

Castillo PE, Carleton A, Vincent JD, Lledo PM (1999) Multiple and opposing roles of cholinergic transmission in the main olfactory bulb. J Neurosci 19:9180-9191.

Chaput M, Holley A (1979) Spontaneous activity of olfactory bulb neurons in awake rabbits, with some observations on the effects of pentobarbital anaesthesia. J Physiol (Paris) 75:939-948.

Chaput MA (1986) Respiratory-phase-related coding of olfactory information in the olfactory bulb of awake freely-breathing rabbits. Physiol Behav 36:319-324.

Fee MS, Leonardo A (2001) Miniature motorized microdrive and commutator system for chronic neural recording in small animals. J Neurosci Methods 112:83-94.

Fontanini A, Bower JM (2005) Variable coupling between olfactory system activity and respiration in ketamine/xylazine anesthetized rats. J Neurophysiol 93:3573-3581.

Haberly LB (2004) Olfactory cortex. In: The synaptic organization of the brain (Shepherd GM, ed), pp 415-454. New York: Oxford UP.

Hardy A, Palouzier-Paulignan B, Duchamp A, Royet JP, Duchamp-Viret P (2005) 5-Hydroxytryptamine action in the rat olfactory bulb: in vitro electrophysiological patch-clamp recordings of juxtaglomerular and mitral cells. Neuroscience 131:717-731.

Igarashi KM, Mori K (2005) Spatial representation of hydrocarbon odorants in the ventrolateral zones of the rat olfactory bulb. J Neurophysiol 93:1007-1019.

Johnson BA, Farahbod H, Saber S, Leon M (2005) Effects of functional group position on spatial representations of aliphatic odorants in the rat olfactory bulb. J Comp Neurol 483:192-204.

Kasa P, Hlavati I, Dobo E, Wolff A, Joo F, Wolff JR (1995) Synaptic and non-synaptic cholinergic innervation of the various types of neurons in the main olfactory bulb of adult rat: immunocytochemistry of choline acetyltransferase. Neuroscience 67:667-677.

Kashiwadani H, Sasaki YF, Uchida N, Mori K (1999) Synchronized oscillatory discharges of mitral/tufted cells with different molecular receptive ranges in the rabbit olfactory bulb. J Neurophysiol 82:1786-1792. 
Kauer JS (2002) On the scents of smell in the salamander. Nature 417:336-342.

Kay LM, Laurent G (1999) Odor- and context-dependent modulation of mitral cell activity in behaving rats. Nat Neurosci 2:1003-1009.

Lee MG, Hassani OK, Alonso A, Jones BE (2005) Cholinergic basal forebrain neurons burst with theta during waking and paradoxical sleep. J Neurosci 25:4365-4369.

Le Jeune H, Jourdan F (1993) Cholinergic innervation of olfactory glomeruli in the rat: an ultrastructural immunocytochemical study. J Comp Neurol 336:279-292.

Le Jeune H, Jourdan F (1994) Acetylcholinesterase-containing intrinsic neurons in the rat main olfactory bulb: cytological and neurochemical features. Eur J Neurosci 6:1432-1444.

Lennie P (2003) The cost of cortical computation. Curr Biol 13:493-497.

Lledo PM, Gheusi G, Vincent JD (2005) Information processing in the mammalian olfactory system. Physiol Rev 85:281-317.

Luo M, Katz LC (2001) Response correlation maps of neurons in the mammalian olfactory bulb. Neuron 32:1165-1179.

Macrides F, Chorover SL (1972) Olfactory bulb units: activity correlated with inhalation cycles and odor quality. Science 175:84-87.

Manns ID, Alonso A, Jones BE (2000) Discharge properties of juxtacellularly labeled and immunohistochemically identified cholinergic basal forebrain neurons recorded in association with the electroencephalogram in anesthetized rats. J Neurosci 20:1505-1518.

Margrie TW, Brecht M, Sakmann B (2002) In vivo, low-resistance, wholecell recordings from neurons in the anaesthetized and awake mammalian brain. Pflügers Arch 444:491-498.

Motokizawa F (1996) Odor representation and discrimination in mitral/ tufted cells of the rat olfactory bulb. Exp Brain Res 112:24-34.

Moulton DG (1963) Electrical activity in the olfactory system of rabbits with indwelling electrodes. In: Olfaction and taste I (Zotterman Y, ed), pp 71-84. Oxford: Pergamon.

Movshon JA, Albright TD, Stoner GR, Majaj NJ, Smith MA (2003) Cortical responses to visual motion in alert and anesthetized monkeys. Nat Neurosci 6:3-4.

Nagayama S, Takahashi YK, Yoshihara Y, Mori K (2004) Mitral and tufted cells differ in the decoding manner of odor maps in the rat olfactory bulb. J Neurophysiol 91:2532-2540.

Nickell WT, Shipley MT (1988) Two anatomically specific classes of candidate cholinoceptive neurons in the rat olfactory bulb. J Neurosci 8:4482-4491.

Nicoll RA (1972) The effects of anesthetics on synaptic excitation and inhibition in the olfactory bulb. J Physiol Lond 223:803-814.

Olshausen BA, Field DJ (2004) Sparse coding of sensory inputs. Curr Opin Neurobiol 14:481-487.

Onoda N, Mori K (1980) Depth distribution of temporal firing patterns in olfactory bulb related air-intake cycles. J Neurophysiol 44:29-39.
Pack CC, Berezovskii VK, Born RT (2001) Dynamic properties of neurons in cortical area MT in alert and anaesthetized macaque monkeys. Nature 414:905-908.

Pager J (1978) Ascending olfactory information and centrifugal influxes contributing to a nutritional modulation of the rat mitral cell responses. Brain Res 140:251-269.

Pager J (1983) Unit responses changing with behavioral outcome in the olfactory bulb of unrestrained rats. Brain Res 289:87-98.

Pager J (1985) Respiration and olfactory bulb unit activity in the unrestrained rat: statements and reappraisals. Behav Brain Res 16:81-94.

Paxinos G, Franklin KBJ (2003) The mouse brain in stereotaxic coordinates, Ed 2. San Diego: Academic.

Rinberg D, Gelperin A (2006) Olfactory neuronal dynamics in behaving animals. Semin Cell Dev Biol, in press.

Salcedo E, Zhang CB, Kronberg E, Restrepo D (2005) Analysis of traininginduced changes in ethyl acetate odor maps using a new computational tool to map the glomerular layer of the olfactory bulb. Chem Senses 30:615-626.

Schmidt MF, Konishi M (1998) Gating of auditory responses in the vocal control system of awake songbirds. Nat Neurosci 1:513-518.

Scott JW, Stewart WB (1979) Mechanisms of augmented field potential responses in the rat olfactory bulb. Brain Res 163:21-31.

Shipley MT, Ennis M (1996) Functional organization of olfactory system. J Neurobiol 30:123-176.

Sobel EC, Tank DW (1993) Timing of odor stimulation does not alter patterning of olfactory bulb unit activity in freely breathing rats. J Neurophysiol 69:1331-1337.

Stewart WB, Scott JW (1976) Anaesthetic-dependent field potential interactions in the olfactory bulb. Brain Res 103:487-499.

Uchida N, Mainen ZF (2003) Speed and accuracy of olfactory discrimination in the rat. Nat Neurosci 6:1224-1229.

Wachowiak M, Cohen LB (2001) Representation of odorants by receptor neuron input to the mouse olfactory bulb. Neuron 32:723-735.

Wellis DP, Scott JW (1990) Intracellular responses of identified rat olfactory bulb interneurons to electrical and odor stimulation. J Neurophysiol 64:932-947.

Wilson DA, Sullivan RM, Gall CM, Guthrie KM (1996) NMDA-receptor modulation of lateral inhibition and c-fos expression in olfactory bulb. Brain Res 719:62-71.

Xu F, Greer CA, Shepherd GM (2000) Odor maps in the olfactory bulb. J Comp Neurol 422:489-495.

Xu F, Liu N, Kida I, Rothman DL, Hyder F, Shepherd GM (2003) Odor maps of aldehydes and esters revealed by functional MRI in the glomerular layer of the mouse olfactory bulb. Proc Natl Acad Sci USA 100: 11029-11034. 